\title{
Preisach model of hysteresis for the Piezoelectric Actuator Drive
}

\author{
Zsurzsan, Tiberiu-Gabriel; Andersen, Michael A. E.; Zhang, Zhe; Andersen, Nils Axel
}

Published in:

Proceedings of the 41st Annual Conference of the IEEE Industrial Electronics Society

Publication date:

2015

Document Version

Peer reviewed version

Link back to DTU Orbit

Citation (APA):

Zsurzsan, T-G., Andersen, M. A. E., Zhang, Z., \& Andersen, N. A. (2015). Preisach model of hysteresis for the Piezoelectric Actuator Drive. In Proceedings of the 41st Annual Conference of the IEEE Industrial Electronics Society IEEE.

\section{General rights}

Copyright and moral rights for the publications made accessible in the public portal are retained by the authors and/or other copyright owners and it is a condition of accessing publications that users recognise and abide by the legal requirements associated with these rights.

- Users may download and print one copy of any publication from the public portal for the purpose of private study or research.

- You may not further distribute the material or use it for any profit-making activity or commercial gain

- You may freely distribute the URL identifying the publication in the public portal

If you believe that this document breaches copyright please contact us providing details, and we will remove access to the work immediately and investigate your claim. 


\title{
Preisach model of hysteresis for the Piezoelectric Actuator Drive
}

\author{
Tiberiu-Gabriel Zsurzsan, Michael A.E. Andersen, Zhe Zhang, Nils A. Andersen \\ DTU Electrical Engineering \\ Technical University of Denmark \\ Oersteds Plads, bld. 349 \\ Kgs. Lyngby, Denmark \\ Email: tgzsur@elektro.dtu.dk
}

\begin{abstract}
The Piezoelectric Actuator Drive (PAD) is a precise piezoelectric motor generating high-torque rotary motion, which employs piezoelectric stack actuators in a wobblestyle actuation to generate rotation. The piezoelectric stacked ceramics used as the basis for motion in the motor suffer from hysteretic nonlinearities. In order to model these nonlinearities, the first-order hysteresis reversal curves of the actuators are measured and a discrete Preisach model is derived. This forms a basis that enables the study of different compensation methods. The results show matching between measured and estimated responses within $95.8 \%$
\end{abstract}

Keywords - motor, actuator, piezoelectric, stack, multilayer, hysteresis, model, Preisach

\section{INTRODUCTION}

The Piezoelectric Actuator Drive (PAD) shown in Fig. 1 is a type of rotary motor that transforms the linear motion of piezoelectric stack actuators into a precise rotation [1]. The operating principle, illustrated in Fig. 2, is based on a wobblestyle motor, where the motor ring is actuated into an off-center circular motion around an axially constrained shaft, thereby generating rotational motion. A micro-mechanical toothing interface is machined between the ring and shaft, as shown in Fig. 3. This enables both high positioning accuracy and output torque [2]. The actuation method, combined with the type of toothing and the inherent large stiffness of the piezoelectric stack actuators makes the PAD appropriate for open-loop control [3].

By construction, the PAD motor behaves like a fully capacitive rotary machine, due to the piezoelectric stack actuators it employs. These actuators exhibit desirable properties such as large stiffness and a reversibility of the piezoelectric effect, enabling them to act as both actuators and sensors [4]. Nevertheless, two dominating nonlinearities persist in the control of piezoelectric-based actuation, namely hysteresis and creep [5]. Of the two effects, hysteresis has a much larger impact on the positioning precision of the motor, especially under dynamic operation. The purpose of this paper is to model this effect in order to correctly predict the response of the PAD.

Modeling hysteresis in the piezoelectric motor is a necessary step in developing compensation methods to negate the effects. The most encountered method of modeling hysteresis in literature remains the Preisach-Krasnoselskii scalar model [6], which provides a straightforward method of representing the hysteretic effect. Although initially developed for the purpose

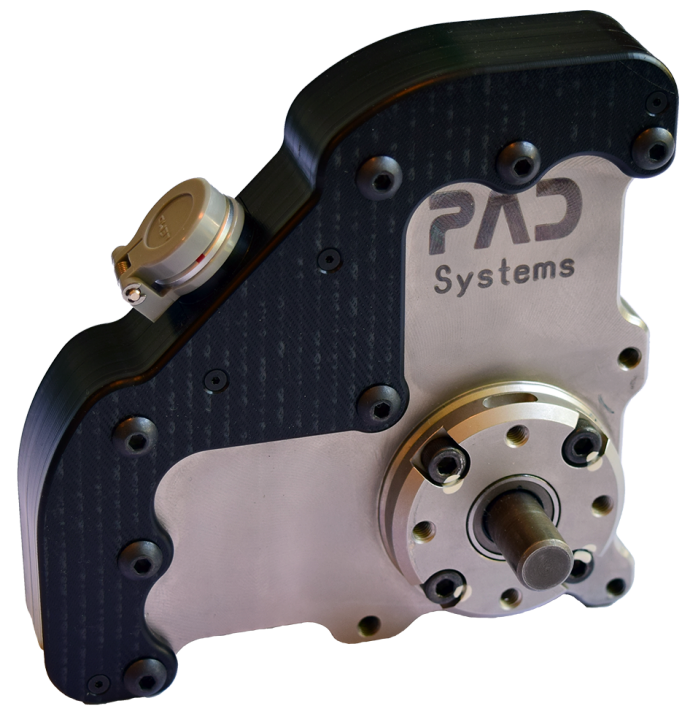

Fig. 1: The Piezoelectric Actuator Drive (PAD)

of modeling magnetic hysteresis, due to its fenomenological nature, the method can be used to model any type of hysteresis [7]. This fact, combined with the generally predictable performance has led the Preisach model to be the preferred method used when hysteresis is involved.

Many modifications and improvements to the original model have been proposed, such as accounting for dynamic input changes [8], [9] or reducing the computational complexity by simplifying the parameter identification process [10]. For this paper, simplicity and real-time execution are the most important factors in determining the method used. Therefore, the classical model enhanced with Mayergoyz's observations on determining the Everett function from the first-order inverting loops [6], [11] is chosen. The paper starts by presenting the underlying principle of the classical Preisach model in section II, followed by a short description of the experimental set-up and tests performed in section III. The model response is then compared to experimental measurements in Section IV, after which the conclusion is presented in section V.

\section{The Classical Preisach Model}

The idea behind the classical scalar Preisach model consists of describing the hysteretic effect through the use of an 


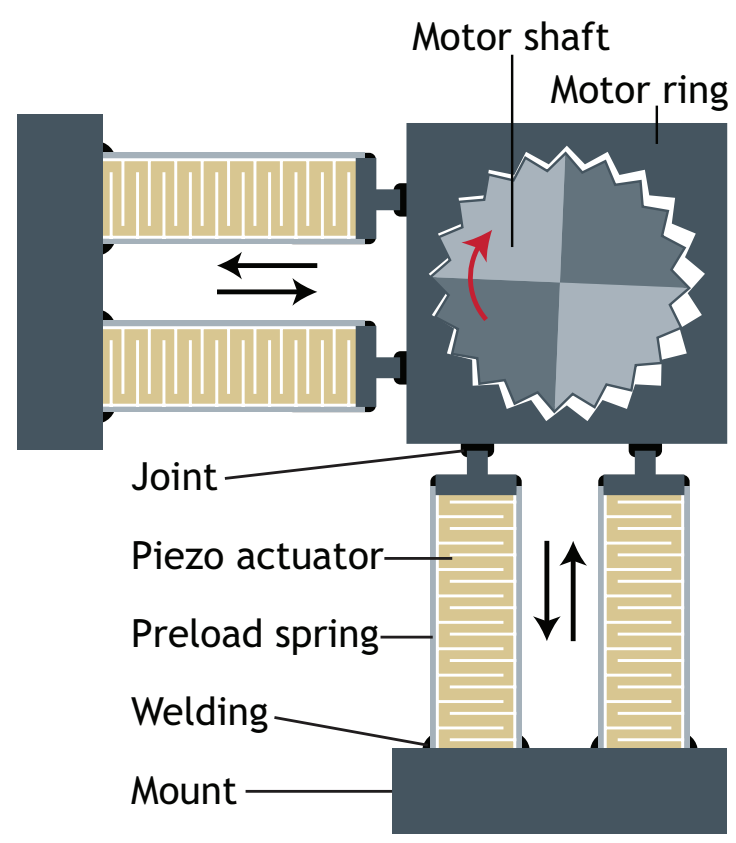

Fig. 2: PAD operating principle

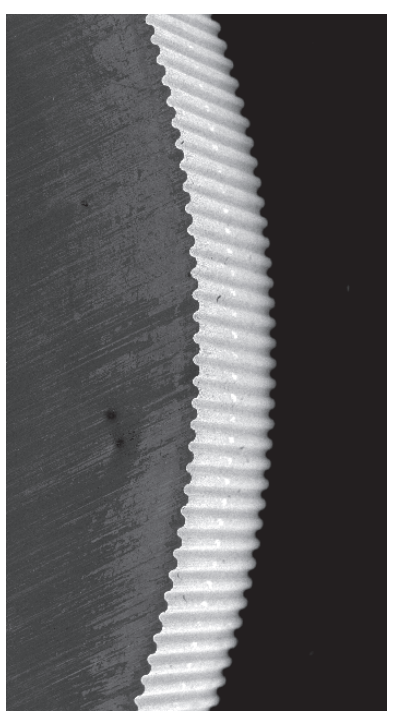

Fig. 3: Microtoothing on the motor shaft. Tooth distance is $120 \mu \mathrm{m}$ and depth is $38 \mu \mathrm{m}$

infinite number of parallel-connected relay-type two-state discontinuous operators $\gamma_{\alpha \beta}[u(t)]$, called basic hysterons. These elements represent relay-like loops on the input-output plane with two values, $\alpha$ and $\beta$, acting as the 'on' and 'off' thresholds, with $\beta \leq \alpha$. When the input signal $u(t)$ becomes greater than $\alpha$, the output of the operator becomes 'high' and goes to 'low' state only when the input becomes less than $\beta$. In between tresholds, the previous value is retained. More explicitly, in the case of piezoelectric devices this can be written mathematically as

$$
\gamma_{\alpha \beta}[u(t)]= \begin{cases}0 & u(t) \leq \beta \\ \gamma_{\alpha \beta}[u(t)] & \beta \leq u(t) \leq \alpha \\ 1 & u(t) \geq \alpha\end{cases}
$$

This representation of the basic hysteretic operator, repre-

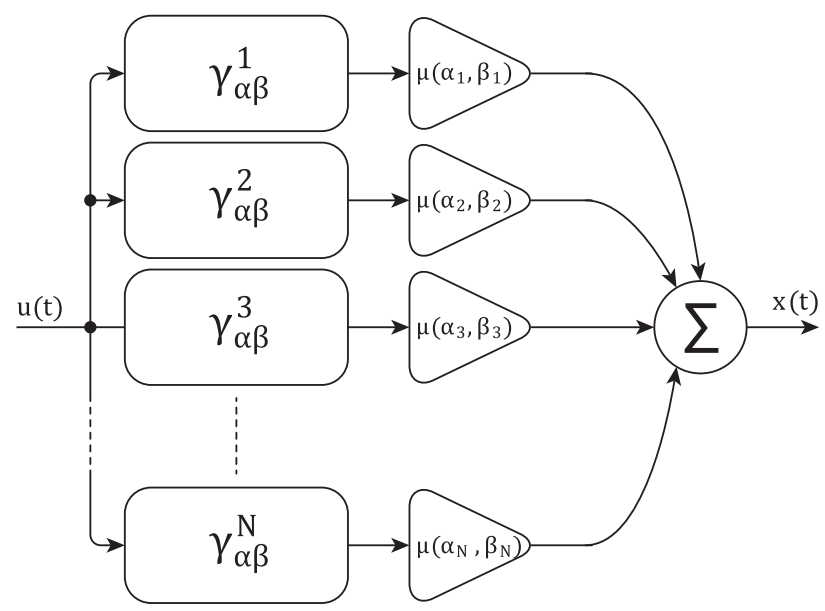

Fig. 4: Parallel connection of elementary Preisach hysterons with weighting gains

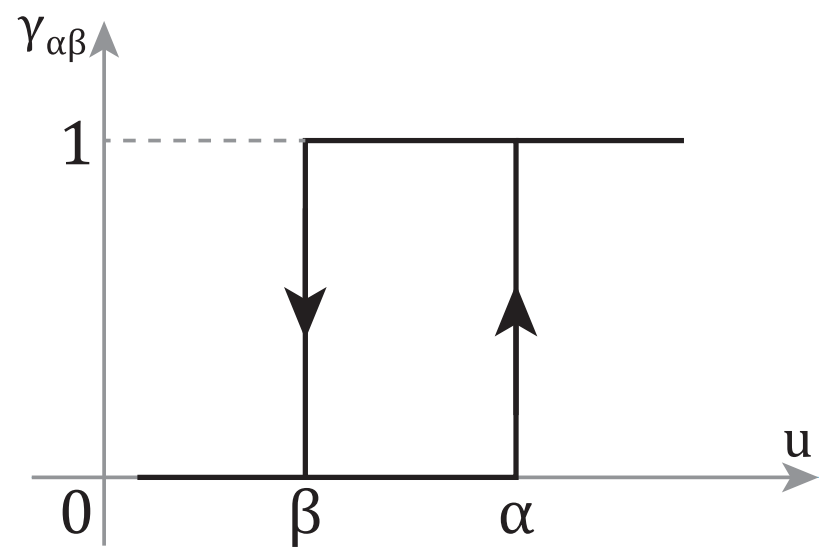

Fig. 5: The elementary Preisach hysteron with thresholds $\alpha$ and $\beta$. The arrows represent one-way state transitions

sented graphically in Fig. 5, is in agreement with the property of a hsyteretic system where the output depends not only on the input, but past values of the input as well. In other words, hysteresis is a nonlinearity with a non-local memory effect.

In order to be able to reproduce a wide range of hysteresis curves, the outputs of the basic hysterons are then weighted with an experimentally-determined weighting function $\mu(\alpha, \beta)$. Determining this function represents the major challenge in deriving and using the Preisach hysteresis model.

The structural block diagram of the Preisach model is shown in Fig. 4. It illustrates the parallel connection and weighting of individual elementary hysterons, whose sum produces the instantaneous output. Mathematically, this is expressed as

$$
x(t)=\iint_{\alpha \geq \beta} \mu(\alpha, \beta) \gamma_{\alpha \beta}[u(t)] \mathrm{d} \alpha \mathrm{d} \beta
$$

where $u(t)$ is the voltage input to the piezoelectric actuator, $x(t)$ represents the output displacement, $\mu(\alpha, \beta)$ is the experimentally-obtained weighting function and $\gamma_{\alpha \beta}[u(t)]$ is the hysteron state.

A geometrical interpretation of the hysteron plane (also called the $\alpha-\beta$ plane) greatly facilitates the understanding of (2) and the Preisach model in general. In this plane, a so-called 
Preisach triangle $T_{0}$ is defined, which represents the region of operation of the actuator, bordered by $\alpha_{\max }, \beta_{\max }, \alpha_{\min }$ and $\beta_{\text {min }}$. Only the surface above the diagonal given by $\alpha=\beta$ has any physical meaning and therefore $T_{0}$ is an upper triangular surface. The elementary hysterons $\gamma_{\alpha \beta}[u(t)]$ have a direct correlation to the $\alpha-\beta$ half-plane in such a way that at any point in time $T_{0}$ is divided into two surfaces $S^{+}$and $S^{-}$, representing the $(\alpha, \beta)$ pairs for which $\gamma_{\alpha \beta}[u(t)]=1$ or 0 , respectively. This is in stark contrast to the method being applied to describe magnetic hysteresis, where in order to wipe out the magnetization, a negative input has to be applied. In other words, in piezoelectric phenomena a lack of excitation field results in a zero net output.

Thereby, for a monotonic increase of the input $u(t)$, the input-output plane shows an ascending hysteresis branch, while the $\alpha-\beta$ half-plane 'fills up' from the bottom to the horizontal line defined by $\alpha=\left\{\alpha_{1} \mid \alpha_{1} \leq u(t)\right\}$. Similarly, a monotonic decrease in input will then determine the surface to 'empty', but this process is orthogonal to the one for increasing input. Therefore the 'filled' space $T_{0}$ will empty starting from the right towards the vertical line defined by $\beta=\left\{\beta_{1} \mid \beta_{1} \geq u(t)\right\}$. Thereby a stochastic input signal with several extrema will be represented as a combination of 'filled' and 'emptied' areas on the triangle, delimited by a boundary staircase layer, denoted $L$. The problem then boils down to finding the area under the obtained staircase curve. This process is visually exemplified in Fig. 6.

An important property of the Preisach model is the wipeout feature, whereby the input history is erased when the input increases above (or decreases below) the previous points of extrema. Specifically, in Fig. $6 \mathrm{~d}$ if the input were to increase beyond $\alpha_{1}$, then the pair $\left(\alpha_{1}, \beta_{2}\right)$ would be removed from the history. Visually, the corner represented by $\left(\alpha_{1}, \beta_{2}\right)$ on the half-plane would be 'engulfed' by the vertically increasing $S^{+}$ surface. In time domain, this coincides with $u(t)$ increasing in amplitude beyond its previous extremum. This feature is important in order to prevent excessive history growth.

Using the presented geometric interpretation, the Preisach model (2) can be rewritten as

$x(t)=\iint_{S^{+}} \mu(\alpha, \beta) \gamma_{\alpha \beta}[u(t)] \mathrm{d} \alpha \mathrm{d} \beta+\iint_{S^{-}} \mu(\alpha, \beta) \gamma_{\alpha \beta}[u(t)] \mathrm{d} \alpha \mathrm{d} \beta$

Considering that in the case of piezoelectric actuators $\gamma_{\alpha \beta}[u(t)]=\left.0\right|_{u(t) \leq \beta}$ and $\gamma_{\alpha \beta}[u(t)]=\left.1\right|_{u(t) \geq \alpha}$, (3) can be reduced to

$$
x(t)=\iint_{S^{+}} \mu(\alpha, \beta) \mathrm{d} \alpha \mathrm{d} \beta
$$

The next step in completing the Preisach hysteresis model is determining the $\mu(\alpha, \beta)$ weighting function. This is done more easily by using the system's first-order reversal curves, shown in Fig. 7a, to construct the describing Everett function [12], thereby eliminating the computationally-intensive double integration. A term denoted with $x_{\alpha}$ is defined as the piezoelectric expansion on the limiting ascending branch of the hysteresis curve corresponding to an input value of $\alpha$ and, similarly, $x_{\alpha \beta}$ is defined as the expansion on the descending branch that starts at the previous $x_{\alpha}$. Hereby, the Everett
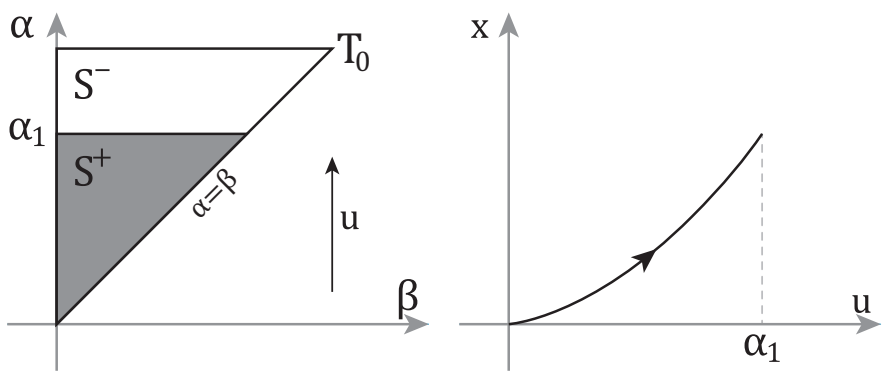

(a)
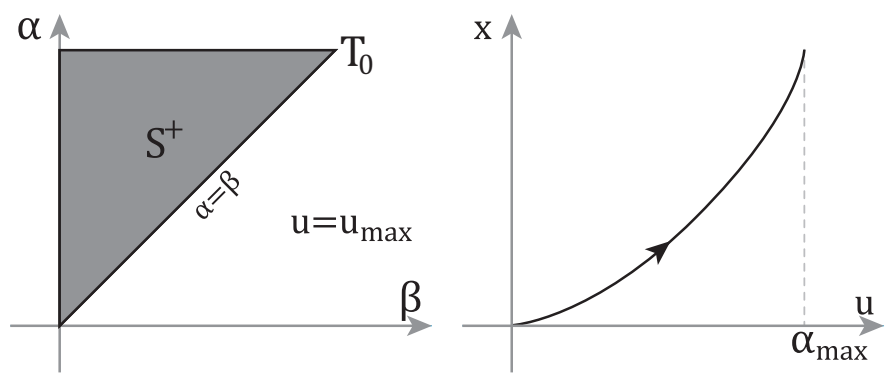

(b)
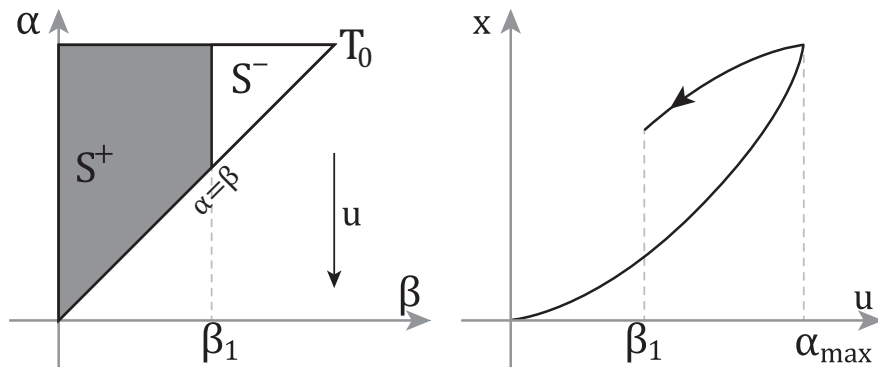

(c)
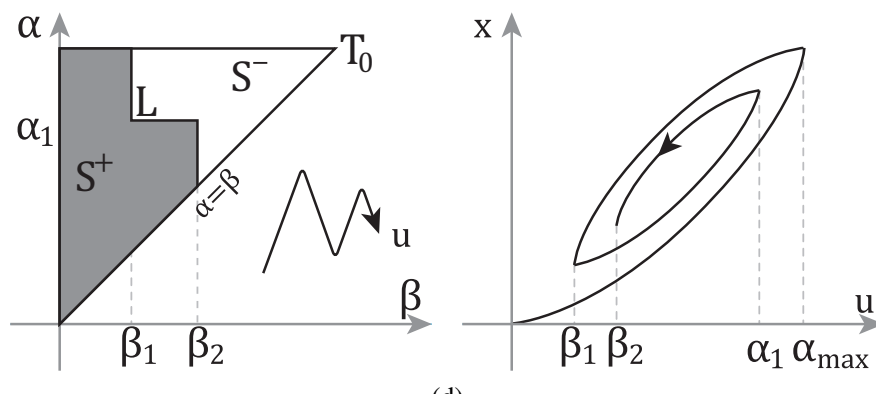

(d)

Fig. 6: Representation of the Preisach triangle, with examples for increasing (a), maximum (b), decreasing (c) and progressively decreasing amplitude (d) triangular input and their respective surface divisions.

function is defined as

$$
X(\alpha, \beta)=\frac{1}{2}\left(x_{\alpha}-x_{\alpha \beta}\right)
$$

By choosing two arbitrary points $x_{\alpha}$ and $x_{\alpha \beta}$ as shown in Fig. $7 \mathrm{~b}$ and expressing them in terms of Preisach equation, the geometric interpretation of the Everett function shows that:

$$
x_{\alpha}=\iint_{S^{+}+T_{1}} \mu(\alpha, \beta) \mathrm{d} \alpha \mathrm{d} \beta
$$




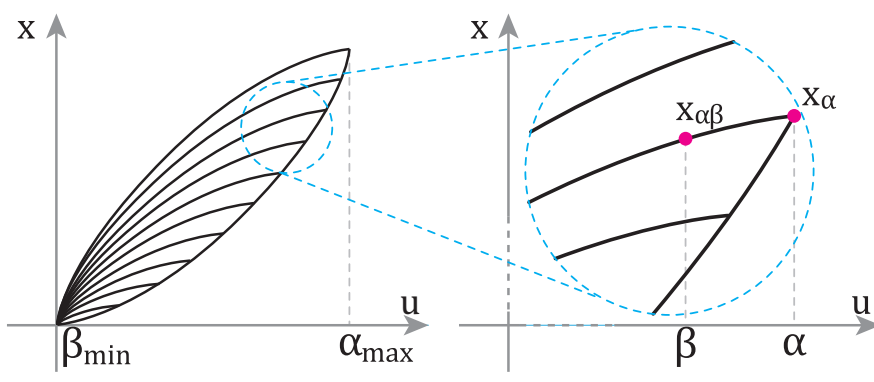

(a)
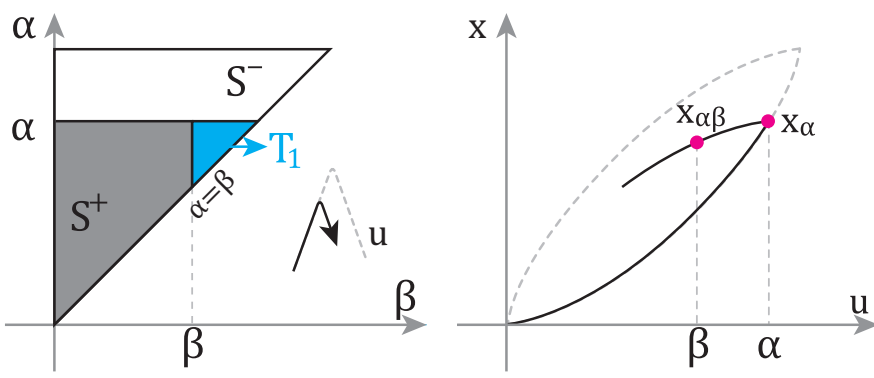

(b)

Fig. 7: First-order reversal curves used to construct the Everett function (a) and an example of obtaining the function together with the graphical interpretation (b). The surface $T_{1}$ represents the difference in $S^{+}$when the input decreases from $\alpha$ to $\beta$.

$$
x_{\alpha \beta}=\iint_{S^{+}} \mu(\alpha, \beta) \mathrm{d} \alpha \mathrm{d} \beta
$$

Now by rewriting (5) and taking (7) and (6) into account while considering that the double integral over the total surface $S^{+}+$ $T_{1}$ is equal to the sum of the integrals over each surface, $X(\alpha, \beta)$ becomes:

$$
X(\alpha, \beta)=\frac{1}{2} \iint_{T_{1}} \mu(\alpha, \beta) \mathrm{d} \alpha \mathrm{d} \beta
$$

This shows that the Everett function represents the double integral of the weighting function over a component surface. Finding the actual weighting function $\mu(\alpha, \beta)$ requires a double differentiation of the measured sum of Everett functions, but doing that with noisy, experimental data introduces even more noise and might render the result unusable. Therefore, it is more convenient to now introduce the discrete Preisach model and re-express the initial formulation shown in (2) to include the Everett terms [8]. By considering an $N$ number of memorized extrema, the piezoelectric expansion becomes:

$$
x(t)= \begin{cases}X\left(u(t), \beta_{N}\right)+ & \\ +\sum_{i=1}^{N}\left(X\left(\alpha_{i}, \beta_{i-1}\right)-X\left(\alpha_{i}, \beta_{i}\right)\right) & \dot{u}(t)>0 ; \\ X\left(\alpha_{N}, \beta_{N-1}\right)-X\left(\alpha_{N}, u(t)\right)+ & \\ +\sum_{i=1}^{N}\left(X\left(\alpha_{i}, \beta_{i-1}\right)-X\left(\alpha_{i}, \beta_{i}\right)\right) & \dot{u}(t)<0 .\end{cases}
$$

In order to obtain the values needed for each Everett function,
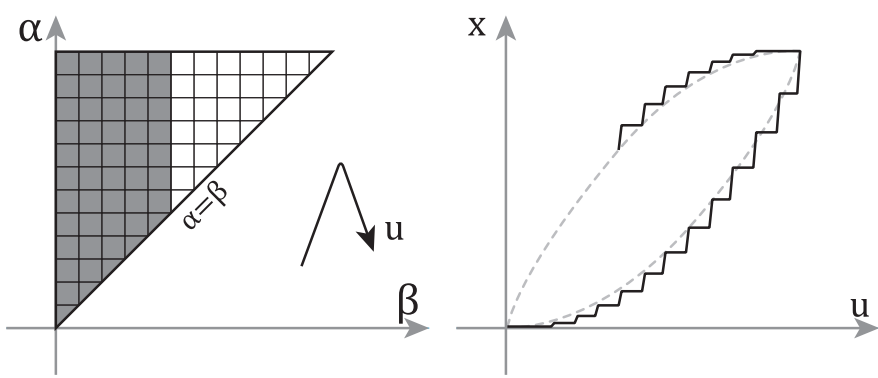

Fig. 8: Division of the Preisach plane into $N=12$ equally-distanced values for $\alpha$ and $\beta$, and the resulting 12 discrete levels obtained on the hysteresis curve.

and consequently the discrete Preisach model, the previouslydefined limiting triangle $T_{0}$ is now split up into a mesh containing values for the point pairs $\left\{\left(\alpha_{i}, \beta_{j}\right) \mid i=1 . . N, j=i . . N\right\}$. Fig. 8 shows the geometrical interpretation of the discrete Preisach plane on the left hand side, together with the obtained input-output representation on the right-hand side, for an arbitrary value of $N=12$. The number of $\alpha$ and $\beta$ values equals the number of discrete steps on the hysteresis curve. Naturally, the higher $N$ is chosen, the finer the mesh becomes and consequently, the finer the hysteresis representation will become. This is at the expense of exponentially increasing memory requirements to store all the values.

\section{EXPERIMENTAL SET-UP}

Through (10), a theoretical basis for modeling hysteresis in piezoelectric actuators is set. The PAD motor employs four piezoelectric stack actuators in order to generate rotational motion, as presented in Fig. 2. In order to be able to model the effects of hysteresis in the motor, the first-order reversal curves of these actuators have to be measured and the corresponding Preisach plane needs to be created. To accomplish this, an experimental set-up that can measure these parameters was built.

The experimental setup is presented in Fig. 9 and consists of a computer with a dSPACE single-board PCI system, a linear voltage amplifier and a laser displacement sensor. The laser sensor is a Keyence LC-2440 sensor head connected to a Keyence LC-2400 controller. The measurement system has measuring range of $\pm 3 \mathrm{~mm}$, maximum resolution of $0.2 \mu \mathrm{m}$ and a bandwidth of $20 \mathrm{kHz}$. The unit under test is a Noliac custom piezoelectric stack actuator laser-welded into a springload casing with $750 \mathrm{~N}$ preloading. The actuator is rated for $200 \mathrm{~V}$ input voltage, producing a maximum elongation of $60 \mu \mathrm{m}$.

A Matlab script is used to produce a positively biased receding sawtooth waveform which is then amplified and drives the actuator. The waveform is adjustable in time, envelope slope and number of teeth. This type of waveform is chosen in order to eliminate the effects of creep while keeping all hysteresis information intact. Data acquisition of the position value is then performed and all data is stored into Matlab for processing.

\section{Measurements And Results}

In order to identify the Preisach model and build the $\alpha-\beta$ plane, the first-order reversal curves are measured for a 


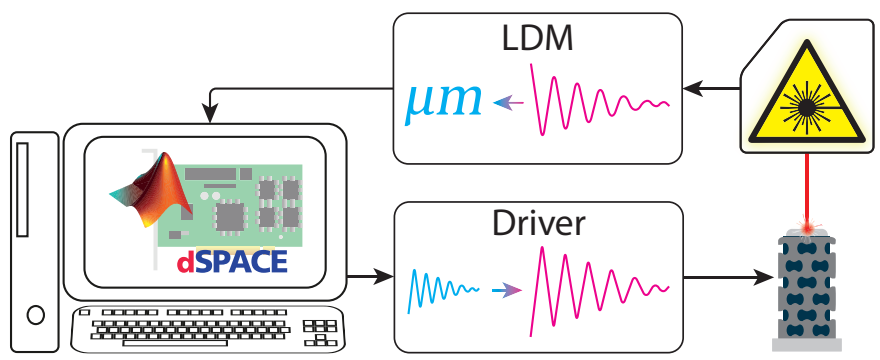

Fig. 9: Block diagram of the experimental set-up. A computer equipped with a PCI dSPACE board is used. The signal generated is amplified through a voltage amplifier. The unit under test is a Noliac custom piezoelectric stack actuator. Elongation is measured using a Keyence LC-2440 laser sensor connected to a Keyence LC-2400 laser displacement meter (LDM).

single piezoelectric actuator from the four. The other actuators are assumed to be identical with the measured one. This assumption is reasonable, within the fabrication tolerances of the actuators themselves but it will of course impact the model accuracy.

Fig. 10a shows the receding sawtooth voltage signal applied to the stack actuator, as measured from the output of the voltage amplifier. The frequency of the signal is $0.5 \mathrm{~Hz}$, it has a length of 50 periods and a linear recession slope of $-2 \mathrm{~V}$ per period. The low frequency is chosen so that the dynamic properties of the actuator do not affect its response.

The measured displacement of the actuator corresponding to the input voltage is shown in Fig. 10b. It has the same frequency and length as the input signal, but the envelope of the recession shows an exponential response with a subunitary exponent, instead of the linear recession seen in the voltage signal. The glitches present in the signals at around the 45 second mark are the result of the measurement system buffer overflowing and missing some samples. A new set of measurements needs to be carried out with special attention to acquisition buffer size and amount of data.

Nevertheless, the response estimated by the developed model is in good agreement with the measured response. Fig. 11 shows a comparison between the real and modeled displacements for an applied voltage signal swinging between $31 \mathrm{~V}-173 \mathrm{~V}-33 \mathrm{~V}-171 \mathrm{~V}$. This specific input voltage swing was chosen at random out of the ranges for which measurements exist, so as to be able to evaluate the model response. The relative error plot in Fig. 11c illustrates that the model response matches the measurements within $95.8 \%$. Therefore the model correctly estimates the actuator's exponential displacement response as a direct result of the triangular input excitation.

\section{CONCLUSIONS}

This paper models the effects of hysteresis in the piezoelectric stack actuators that make up the Piezoelectric Actuator Drive motor. A discrete Preisach model was chosen due to its popularity as a hysteresis modeling tool, relative simplicity and ease of implementation. The model was built based on the measurement of 50 hysteresis reversal curves, from which the corresponding Everett functions were extracted. Validation against a randomly-chosen input variation was done. Finally, the developed and implemented discrete Preisach model allows estimating the displacement of the stacks within the motor with a relative accuracy of $95.8 \%$ and serves as a good starting point for studying and developing different compensation algorithms that are able to eliminate hysteresis.

Future work will focus both on deriving an inverse model for compensation, as well as studying improvements to the developed model. Increasing the number of reversal curves measured to obtain higher accuracy, as well as investigating the effects of higher frequency dynamics on the model are being considered.

\section{ACKNOWLEDGEMENTS}

The authors would like to thank Noliac A/S for providing a PAD motor to be dissected and investigated, as well as the Danish National Advanced Technology Foundation for financial support.

\section{REFERENCES}

[1] A. Kappel, B. Gottlieb, and C. Wallenhauer, "Piezoelectric actuator drive (pad)," AT-AUTOMATISIERUNGSTECHNIK, vol. 56, no. 3, pp. 128135,2008

[2] J. Rucha, H. Liess, J. Heinzl, A. Kappel, T. Schwebel, and B. Gottlieb, "Applicability of a piezo actuator drive as a sensorless high precision drive," 10th International Conference on New Actuators, 2006.

[3] R. Zeichfussl, B. Gottlieb, C. Wallenhauer, S. Herzig, A. Kappel, and T. Luth, "A method for auto-adjustment of a new piezoelectric drive," in Mechatronics, 2009. ICM 2009. IEEE International Conference on, April 2009, pp. 1-6.

[4] C. Wallenhauer, B. Gottlieb, A. Kappel, T. Schwebel, J. Rucha, and T. Lueth, "Accurate load detection based on a new piezoelectric drive principle employing phase-shift measurement," Journal of Microelectromechanical Systems, vol. 16, no. 2, pp. 344-350, 2007.

[5] T.-G. Zsurzsan, C. Mangeot, M. Andersen, Z. Zhang, and N. Andersen, "Piezoelectric stack actuator parameter extraction with hysteresis compensation," in Power Electronics and Applications (EPE'14-ECCE Europe), 2014 16th European Conference on, Aug 2014, pp. 1-7.

[6] T. Doong and I. D. Mayergoyz, "On numerical implementation of hysteresis models," IEEE Transactions on Magnetics, vol. MAG-21, no. 5, pp. 1853-1855, 1985.

[7] I. Mayergoyz and G. Friedman, "Generalized preisach model of hysteresis," Magnetics, IEEE Transactions on, vol. 24, no. 1, pp. 212-217, Jan 1988.

[8] R. B. Mrad and H. Hu, "Dynamic modeling of hysteresis in piezoceramics," vol. 1, pp. 510-515 vol.1, 2001

[9] X. Wang, V. Pommier-Budinger, Y. Gourinat, and A. Reysset, "A modified preisach model for hysteresis in piezoelectric actuators," Informal Proceedings of the 11th International Workshop of Electronics, Control, Measurement, Signals and Their Application To Mechatronics, Ecmsm 2013, Informal Proc. Int. Workshop Electron., Control, Meas., Signals Their Appl. Mechatronics, Ecmsm, p. 6648956, 2013.

[10] F. Weibel, Y. Michellod, P. Mullhaupt, and D. Gillet, "Real-time compensation of hysteresis in a piezoelectric-stack actuator tracking a stochastic reference," 2008 AMERICAN CONTROL CONFERENCE, VOLS 1-12, pp. 2939-2944, 2008

[11] I. D. Mayergoyz and G. Friedman, "On the integral equation of the vector preisach hysteresis model," IEEE Transactions on Magnetics, vol. MAG-23, no. 5, pp. 2638-2640, 1987.

[12] I. Mayergoyz, "Mathematical-models of hysteresis," IEEE TRANSACTIONS ON MAGNETICS, vol. 22, no. 5, pp. 603-608, 1986. 


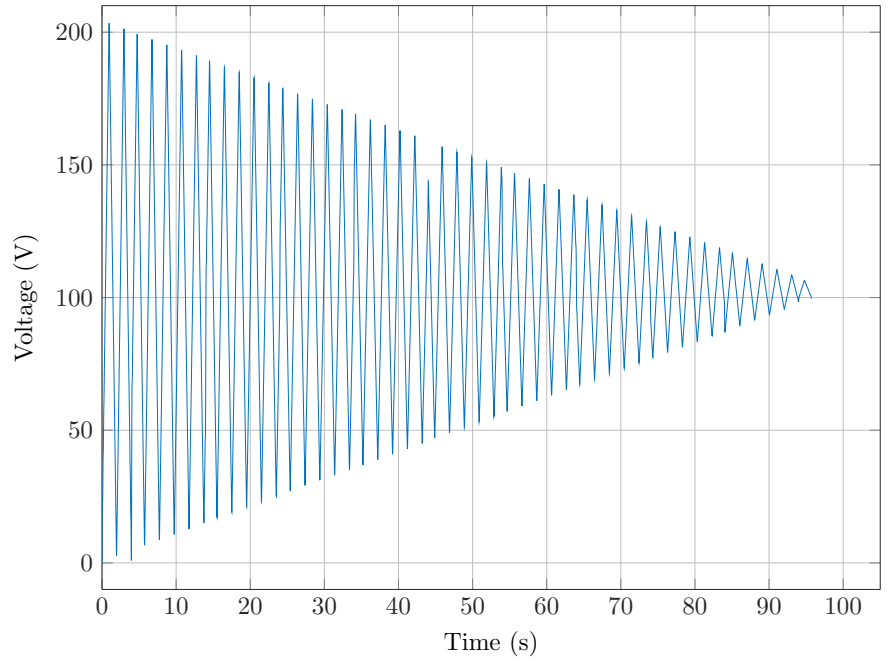

(a)

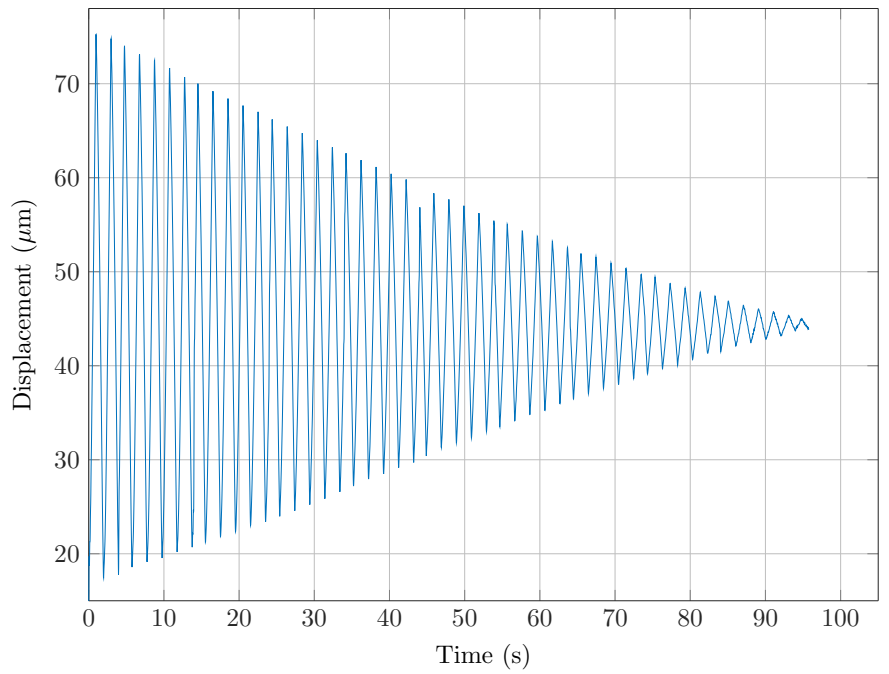

(b)

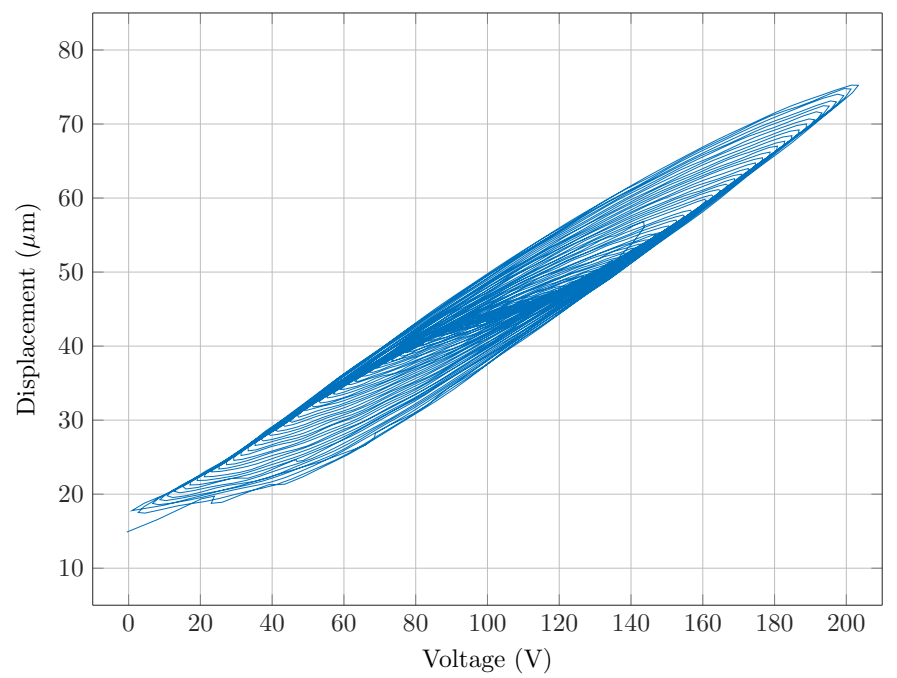

(c)

Fig. 10: Measurement of the first order reversal curves used to extract the model parameters. (a) shows the receding sawtooth signal applied to the piezoelectric stack actuator, measured from the output of the amplifier. (b) is the measured displacement of the piezoelectric actuator. (c) shows the actual reversal curves obtained.

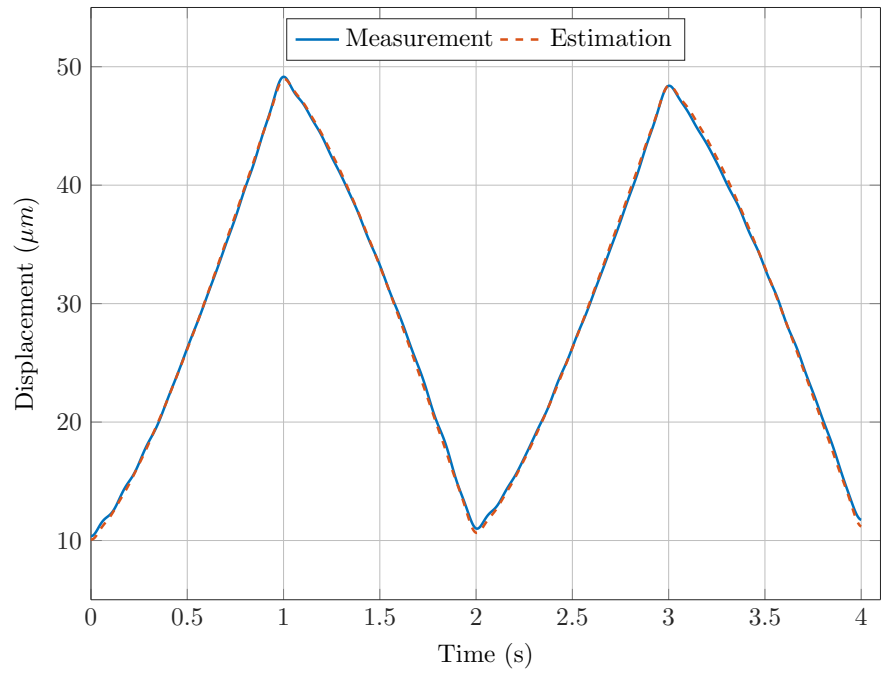

(a)

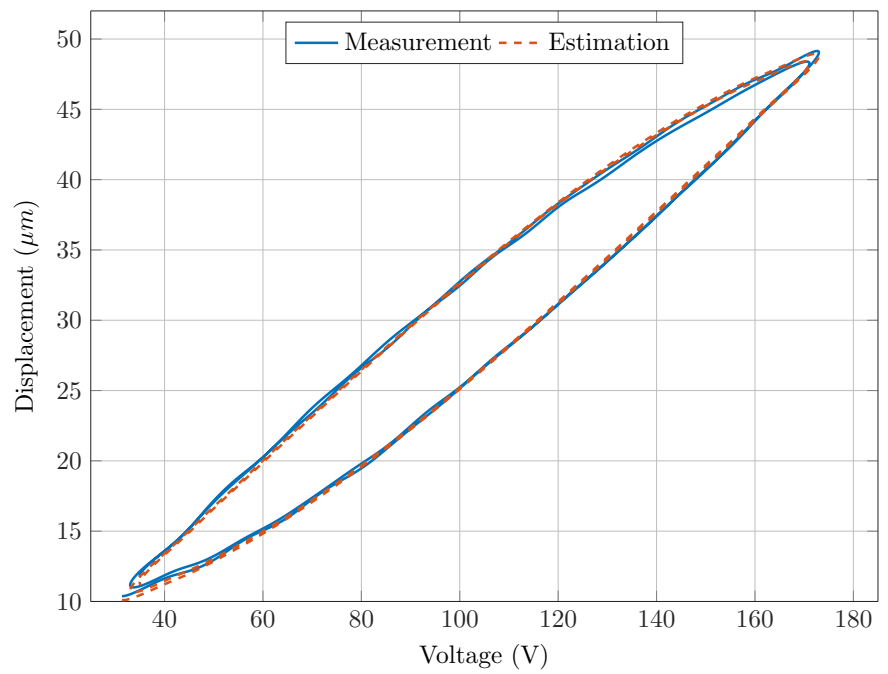

(b)

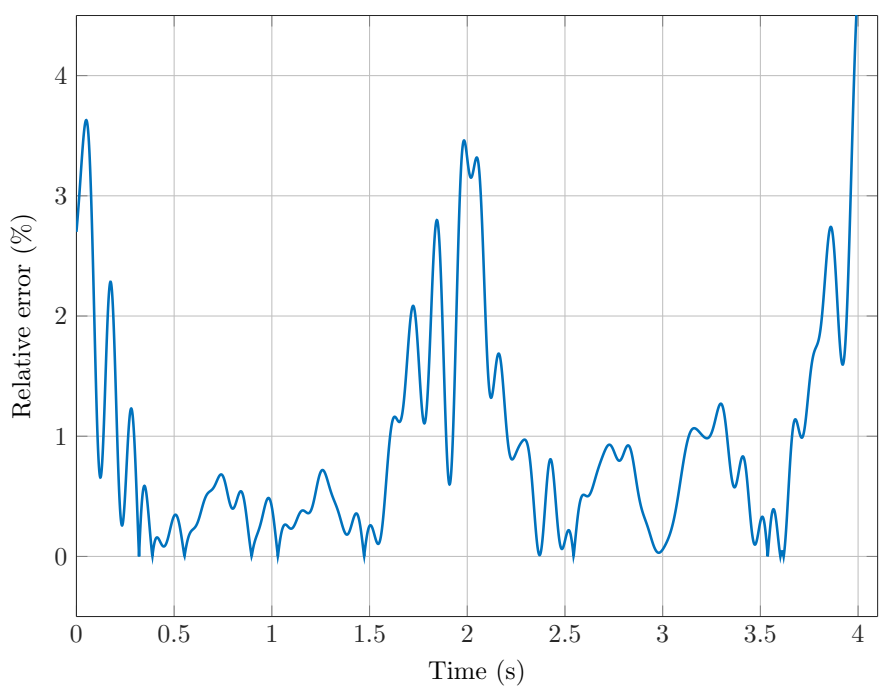

(c)

Fig. 11: Result of the model compared with the actual measured output for an input voltage pattern swinging between $31 \mathrm{~V}-173 \mathrm{~V}-33 \mathrm{~V}-171 \mathrm{~V}$. (a) shows the comparison in time, (b) shows the matching hysteresis curves and (c) contains the relative error plot. 